\title{
Factores psicosociales asociados con violencia de pareja entre hombres homosexuales mexicanos con VIH
}

\section{Psychosocial factors associated with intimate partner violence among Mexican homosexual men with HIV}

\author{
Cecilia Alderete-Aguilarl, Rebeca Robles-García ${ }^{2}$, \\ Luciana Ramos-Lira ${ }^{2}$, Corina Benjet ${ }^{2}$, Nayelli Alvarado-Sánchez ${ }^{3}$ \\ y Marlene Lazcano-Ramírez ${ }^{4}$ \\ Universidad Nacional Autónoma de México ${ }^{1,3}$ \\ Instituto Nacional de Psiquiatría de México "Ramón de la Fuente Muñiz"2 \\ Universidad del Pedregal ${ }^{4}$
}

Autor para correspondencia: Cecilia Alderete A., cecilia.alderete.aguilar@gmail.com.

\section{RESUMEN}

\begin{abstract}
El presente estudio tuvo como objetivo central comparar los factores psicosociales en hombres homosexuales (HH) con y sin violencia de pareja (VP) en una muestra de pacientes de una clínica de atención del VIH en la Ciudad de México. Los resultados revelaron diferencias estadísticamente significativas entre los grupos tanto por sus características individuales como por variables propias de la comunidad gay que parecen evidenciar la asociación entre el rechazo y violencia social hacia minorías sexuales incluyendo la VP en esta población. Los hallazgos sugieren que el fenómeno de la VP entre $\mathrm{HH}$ con VIH requiere el desarrollo de intervenciones específicas para individuos, familias y comunidades.
\end{abstract}

Palabras clave: Violencia de pareja; Hombres; Homosexualidad; VIH; Factores psicosociales.

\begin{abstract}
The main objective of the present study was to compare psychosocial factors of homosexual men (HM) with and without intimate partner violence (IPV) in a sample of patients from an HIV clinic in Mexico City. Statistically significant differences were found between groups regarding both individual characteristics and variables related to the Gay community. Such cultural features seem to associate rejection to social violence within sexual minorities and IPV in this population. These findings suggest that the IPV phenomenon between HM with HIV requires the development of specific interventions for individuals, families and communities.
\end{abstract}

Keywords: Intimate partner violence; Men, Homosexuality; HIV; Psychosocial factors.

\footnotetext{
${ }^{1}$ Programa de Maestría y Doctorado en Ciencias Médicas y de la Salud, Circuito de los Posgrados s/n, Coyoacán, 04510 Ciudad de México, México, tel. (55)40-47-02-20, correo electrónico: cecilia.alderete.aguilar@gmail.com.

${ }^{2}$ Departamento de Investigaciones Epidemiológicas y Psicosociales, Calzada México-Xochimilco 101, Huipulco, Tlalpan, 14370 Ciudad de México, México, tel. (55)41-60-51-43, correos electrónicos: reberobles@imp.edu.mx y ramosl@imp.edu.mx, cbenjet@imp.edu.mx.

${ }^{3}$ Facultad de Estudios Superiores Iztacala, Avenida de los Barrios 1, 54090 Los Reyes Iztacala, Tlalnepantla de Baz, Edo. de México, México, tel. (55)31-29-37-91, correo electrónico: nayelli.alvarado.s@gmail.com.

${ }^{4}$ Departamento de Psicología, Transmisiones 51, Arboledas del Sur, Tlalpan, 14370 Ciudad de México, México, tel. (55)48-66-35-69, correo electrónico: marlene.lazcano@outlook.com.
} 
Recibido: $06 / 01 / 2020$

Aceptado: 22/06/2020

A 1 hablar de violencia de pareja (VP en lo sucesivo) se hace referencia a un patrón de conducta en una relación íntima entre dos personas que genera daño físico, psicológico o sexual (Siemieniuk et al., 2013). Es una conducta agresiva y coercitiva que tiene como propósito controlar a la pareja (Reyes, Rodríguez y Malavé, 2005) y que puede manifestarse mediante agresiones físicas, sexuales, verbales o emocionales, así como por restricciones económicas, aislamiento e intimidación (Siemieniuk et al., 2013).

En general, diferentes autores (Chong, Mak y Kwong, 2013; McClennen, Summers y Vaughan, 2002; Toro y Rodríguez, 2001) han encontrado que las dificultades en la solución de problemas, el uso y abuso de sustancias y la violencia en la familia de origen contribuyen al desarrollo de la violencia en parejas de hombres. Se ha visto también que muchos de los que experimentan violencia con parejas del mismo sexo, al igual que las mujeres, presentan creencias distorsionadas sobre la pareja, por lo que no interpretan sus experiencias como violencia y de hecho minimizan la situación (Siemieniuk et al., 2013).

Aunque se sabe que existen semejanzas, algunos investigadores han señalado diferencias significativas en el contexto en el que ocurre la VP heterosexual y la homosexual (Kimmes et al., 2019; Roberts, 2005), puesto que hay algunos aspectos que son únicos de las parejas del mismo sexo: miedo a revelar la orientación sexual, experiencias previas de violencia y discriminación y falta de recursos adecuados en la comunidad, entre muchos otros (Ard y Makadon, 2011).

Se habla de factores únicos asociados a un tipo de relación que frecuentemente es estigmatizada por la sociedad, lo que probablemente lleva a experimentar prejuicios y discriminación (amenazas verbales, acoso, violencia), internalización de pensamientos negativos sobre la identidad y la orientación sexual, deseo de esconder esta última, o el hecho de ser pareja de alguien del mismo sexo. Todas estas experiencias pueden resultar en un estrés de minoría sexual (Graham, Jensen, Givens, Bowen y Rizo, 2019).

Así, las diferencias en la violencia de pareja en la población heterosexual y en las minorías sexuales pueden ser entendidas usando un marco de referencia de estrés de minoría. Este marco socioecológico promueve el análisis de factores potenciales de riesgo o de protección que los individuos con relaciones con el mismo sexo pueden experimentar individual, relacional, comunitaria y socialmente por el hecho de pertenecer a una minoría sexual (Graham et al., 2019). Entre los factores de riesgo destacan los estresores internalizados (homonegatividad internalizada, ocultación de la orientación sexual) como externalizados (discriminación, rechazo); se ha sugerido que la interacción de factores en los diferentes niveles influye en la experiencia de estrés de las minorías sexuales y como resultado potencia la aparición de la VP (Edwards, Sylaska y Neal, 2015; Kubicek, 2018).

Se ha documentado una alta prevalencia de VP en la población de hombres homosexuales (HH en adelante), equiparable a la de la población heterosexual (Badenes, Sánchez y Longobardi, 2019; Edwards et al., 2015; Rodríguez y Toro, 2005; Tran et al., 2014), con tasas que oscilan entre el 1 y $97 \%$, siendo la violencia psicológica la reportada con mayor frecuencia, en tanto que los estudios sobre la violencia sexual reportan tasas más bajas de victimización (Pimentel, Cheng y Kelly, 2015; Tran et al., 2014).

También se ha documentado una relación estrecha entre la VP y el VIH en hombres (Relf, 2001), con importantes implicaciones para la transmisión del virus, el uso de sustancias y los problemas de salud mental y de salud en general (Pantalone, Rood, Morris y Simoni, 2014); incluso algunos autores sugieren que los tres más grandes problemas que enfrentan los HH son el abuso de sustancias, el VIH y la violencia de pareja (Peterman y Dixon, 2003), y que la interrelación de estas variables genera resultados negativos en la salud de esta población (Pimentel et al., 2015), particularmente vulnerable a los efectos de la VP debido al deterioro significativo en las conductas de autocuidado que esta genera, lo que afecta de forma negativa los marcadores biológicos de la enfermedad (Schafer et al., 
2012). Cabe mencionar que puede haber incluso una falta de apego al tratamiento antirretroviral asociada a la VP y, en consecuencia, una progresión más rápida a la fase de sida y una mayor mortalidad. Así pues, los efectos negativos de la VP en la salud física y mental podrían ser exacerbados en los individuos que además sufren de VIH (Ramachandran, Yonas, Silvestre y Burke, 2010).

La alta prevalencia de la violencia de pareja encontrada en los estudios realizados en HH con VIH indica la importancia de un tamizaje sistematizado para todos los pacientes que se atienden en los centros de VIH, incluidos por supuesto los hombres, a pesar de la percepción común de que solamente las mujeres son las víctimas y los hombres son siempre los agresores (Pantalone, Schneider, Valentine y Simoni, 2012).

La mayoría de las investigaciones sobre la violencia en parejas del mismo sexo se han efectuado en poblaciones angloamericanas, pero se desconoce su gravedad en muchos de los países latinoamericanos. En Puerto Rico, Cuba, Argentina y Chile se han llevado a cabo algunos estudios para conocer la prevalencia y la caracterización de este fenómeno (Ortega, 2014; Reyes et al., 2005; Rodríguez y Toro, 2005: Téllez-Santalla y Walters, 2011; Saldivia, Faundez, Sotomayor y Cea, 2017), aunque no se han encontrado estudios específicos sobre la VP y el VIH en HH. Es por ello que resulta necesario encaminar los esfuerzos para conocer los factores psicosociales asociados con el fenómeno entre los HH latinoamericanos, particularmente entre aquellos con diagnóstico de VIH, en quienes constituye un importante riesgo para su salud.

Así, el presente estudio tuvo como objetivo central comparar algunos factores psicosociales entre HH mexicanos diagnosticados con VIH con y sin VP para detectar diferencias en la frecuencia o magnitud de dichos factores relacionados con la VP ejercida o recibida.

Con base en la revisión de la literatura relativa a los factores de riesgo de VP, tanto en parejas heterosexuales, como aquellos que son específicos en las parejas homosexuales, se planteó la hipótesis de que los HH con VIH que habían ejercido y recibido violencia de pareja mostrarían una frecuencia mayor de antecedentes de violencia intergenera- cional, uso de sustancias, conflictos de pareja (y el consecuente uso de estrategias para afrontarlos), niveles superiores de distorsiones cognitivas (sobre la pareja y el uso de violencia), homonegatividad internalizada y una menor apertura sobre su orientación sexual (outness), en comparación con los HH que no habían experimentado VP.

\section{MÉTODO}

\section{Diseño y participantes}

Se realizó un estudio cuantitativo, no experimental, descriptivo, transversal y comparativo de grupos con y sin VP, conformados mediante muestreo no probabilístico por conveniencia, de HH que acudían a una clínica especializada en detección y atención a personas con VIH (Clínica Especializada Condesa), ubicada en la Ciudad de México. Se incluyeron hombres mayores de 18 años, con diagnóstico confirmado de VIH de seis meses o más que reportaran tener o haber tenido alguna relación de pareja con otro hombre de al menos tres meses de duración, que dicha relación estuviera vigente durante o posteriormente a la confirmación del diagnóstico, que supieran leer y escribir, que aceptaran participar de manera voluntaria y firmaran el formato de consentimiento informado correspondiente. Fueron excluidos aquellos hombres que presentaron alguna alteración de su estado cognitivo (delirium, demencia, psicosis...).

La muestra total del estudio fue de $210 \mathrm{HH}$ con diagnóstico confirmado de VIH, con una edad promedio de 32.15 (D.E. $=8$ ) y una media de años de estudio de 14.32 (D.E. $=2.84$ ), equivalente a estudios universitarios incompletos. De ellos, 76.2\% $(\mathrm{n}=160)$ reportó tener empleo o alguna actividad remunerada; $55.7 \%$ proporcionó datos de una relación actual, mientras que $44.3 \%$ informó sobre su última relación.

En relación con las variables relacionadas específicamente con el VIH, los participantes reportaron que el número de veces en promedio que se realizaron la prueba antes de conocer su diagnóstico fue de 3.45 (D.E. = 3.3); el tiempo promedio en meses que llevaban de conocer su diagnóstico fue de 48.62 (D.E. $=30.2) ; 97.1 \%(\mathrm{n}=204) \mathrm{se}$ 
encontraban tomando tratamiento antirretroviral y $23.3 \%(n=49)$ habían dejado de tomarlo alguna vez desde la confirmación de su diagnóstico, y 76.7\% $(\mathrm{n}=161)$ de los hombres encuestados informó que su pareja conocía su diagnóstico de VIH.

Quienes contestaron el cuestionario reportaron que sus relaciones de pareja con otros hombres habían tenido una duración promedio de 40.3 meses (D.E. $=37.67$, con un rango de 3-243). En una escala de 0 a 10 , el nivel promedio de importancia que asignaron a la relación reportada fue de 8.83 (D.E. $=1.51)$, y 49\% $(\mathrm{n}=103)$ señaló estar viviendo o haber vivido con su pareja.

\section{Variables e instrumentos}

Siguiendo el marco socioecológico del estrés de minoría, se establecieron tres categorías de variables: factores individuales, factores de la relación de pareja y factores de la violencia de pareja. Los primeros incluyeron historia de abuso intergeneracional, uso de sustancias, habilidades para resolución de conflictos (agresividad/asertividad), creencias asociadas con la VP, homonegatividad internalizada, outness (apertura sobre la orientación sexual) y datos sociodemográficos. Los factores de la relación de pareja evaluados fueron duración de la relación, cohabitación y nivel de importancia de la relación.

Los factores de VP abarcaron la percepción de violencia (si el participante consideraba que él, su pareja o ambos habían experimentado violencia en la relación), así como las conductas de violencia física, violencia emocional y violencia sexual, tanto recibida (si el participante había sido receptor de conductas violentas por parte de su pareja), como ejercida (si el participante había ejecutado conductas violentas hacia su pareja).

En una primera etapa, todos los instrumentos fueron adaptados y validados con población mexicana de $\mathrm{HH}$, mostrando propiedades psicométricas adecuadas (Alderete et al., 2017).

\section{Cuestionario de Violencia Doméstica}

para Hombres Gay (Rodríguez y Toro, 2005).

Se utilizaron diferentes escalas de este instrumento para evaluar las conductas de violencia recibida $(\alpha=.910)$, violencia ejercida $(\alpha=.869)$, historia de abuso intergeneracional, uso de sustancias, habilidades para solución de conflictos (asertividad, $\alpha=.829$; agresividad $\alpha=.879$ ), así como información relacionada con el estatus de VIH. Los reactivos se valoran en una escala tipo Likert, con recorrido de 0 a 3, con opciones de respuesta de "nunca" a "frecuentemente", habiendo algunos de opción múltiple (en formato de respuesta Sí/No), lo que permite conocer la presencia y frecuencia de los factores estudiados. Este cuestionario constituye el único instrumento hallado para medir variables asociadas con la VP entre HH latinoamericanos.

\section{Inventario de Pensamientos Distorsionados sobre la Mujer y la Violencia (Echeburúa y Fernández, 1998).}

Se adaptó el lenguaje de dicho inventario para evaluar las creencias distorsionadas relacionadas con la pareja y el uso de la violencia (cf. Ferrer, Bosch, Ramis, Torres y Navarro, 2006). La versión aquí utilizada consta de 19 reactivos que se califican en una escala tipo Likert de 1 a 5, con opciones de respuesta de "Totalmente en desacuerdo a "Totalmente de acuerdo", con coeficiente alfa de Cronbach de 0.792. En él, las puntuaciones más elevadas indican una mayor ocurrencia de creencias distorsionadas.

\section{Inventario de Homonegatividad Internalizada (Mayfield, 2001).}

Se tradujo dicho inventario, diseñado para describir actitudes negativas hacia la homosexualidad en $\mathrm{HH}$ hacia la homosexualidad en general y hacia la propia, cuyos reactivos se evalúan en una escala tipo Likert que va de 1 a 6, con opciones de respuesta "Muy en desacuerdo" a "Muy de acuerdo", y que está dividido en tres subescalas: homonegatividad personal, afirmación gay y moralidad de la homosexualidad. En dicho instrumento, que tiene un coeficiente alfa de Cronbach total de .875 , puntuaciones más altas indican mayor presencia de cada una de estas dimensiones.

Escala de Outness (Frost y Meyer, 2009).

Esta escala consta de cinco preguntas con opciones de respuesta que se valoran en una escala tipo Likert (de 0 a 3, con opciones de respuesta de "Ninguno" a "Todos") para evaluar el nivel de aper- 
tura a la familia, amigos heterosexuales, amigos LGBT, compañeros de trabajo y personal de salud. La versión en español mantuvo los cinco reactivos que originalmente la componen, obteniendo una consistencia interna de 0.855 .

\section{Procedimiento}

Se obtuvo la autorización del Comité de Ética del Instituto Nacional de Psiquiatría "Ramón de la Fuente Muñiz" y de la Clínica Especializada Condesa de la Ciudad de México para poder llevar a cabo la presente investigación en un periodo de un año. Todos los participantes firmaron un formato de consentimiento informado en el cual se les aseguró que su participación sería voluntaria y confidencial, y que podían retirarse de la investigación en cualquier momento, sin que ello generara algún perjuicio para su atención en la clínica.

Se invitó a participar a todos los hombres que acudían a consulta en el servicio de infectología de la clínica, a quienes se les abordaba en las salas de espera, preguntándoles si podían responder una encuesta mientras esperaban su turno de consulta; si respondían de manera afirmativa, se verificaban los criterios de inclusión, se les explicaban los objetivos y procedimientos del estudio, así como el llenado de los instrumentos, y se procedía a la aplicación de los mismos.

\section{Análisis de datos}

Los datos se capturaron y analizaron mediante el paquete estadístico SPSS, versión 20.0, de IBM.
Se definieron las características clínicas y demográficas de la muestra con base en las medias y desviaciones estándar para variables continuas, y con frecuencias y porcentajes para las categóricas. A fin de clarificar la relación entre los factores asociados con la VP, se conformaron dos grupos extremos de comparación: los hombres sin violencia y los hombres con violencia, tanto ejercida como recibida. Después de corroborar los criterios de normalidad mediante la prueba de Kolmogórov-Smirnov, se procedió a comparar las variables entre estos dos grupos extremos con un ANOVA para caracterizar mejor el fenómeno. El nivel alfa prefijado fue, en todos los casos, de 95\%.

\section{RESULTADOS}

Del total de los participantes, $68.1 \%(n=143)$ reportó no haber percibido violencia en su relación de pareja, mientras que $20.48 \%(n=43)$ informó que ambos miembros de la pareja habían sido víctimas de violencia. El porcentaje de hombres que percibieron que solo sus compañeros habían recibido violencia $(2.38 \%, \mathrm{n}=5)$ fue menor que el de aquellos que percibieron que únicamente ellos la habían recibido $(9.05 \%, \mathrm{n}=19)$. La Tabla 1 presenta esta percepción de VP.

La Tabla 2 muestra los tipos de violencia recibida o ejercida por los participantes, ya fuera emocional, física o sexual.

Tabla 1. Percepción de VP.

\begin{tabular}{|l|r|}
\hline \multicolumn{1}{|c|}{ Percepción } & \multicolumn{1}{c|}{$\boldsymbol{n ( \% )}$} \\
\hline No percibió violencia & $143(68.1)$ \\
\hline Solo percibió que él ha sido víctima de violencia & $19(9.05)$ \\
\hline Solo percibió que su compañero ha sido víctima de violencia & $5(2.38)$ \\
\hline Percibió que ambos han sido víctimas de violencia & $43(20.48)$ \\
\hline
\end{tabular}

Tabla 2. Conductas de VP.

\begin{tabular}{|l|r|r|}
\hline \multirow{2}{*}{$\begin{array}{c}\text { Conducta } \\
\text { por tipo de violencia }\end{array}$} & \multicolumn{1}{c|}{ Recibida } & Ejercida \\
\cline { 2 - 3 } & $\boldsymbol{n} \mathbf{( \% )}$ & $\boldsymbol{n}(\mathbf{\%})$ \\
\hline Violencia emocional & $167(79.5)$ & $153(72.9)$ \\
\hline Violencia física & $84(40)$ & $79(37.6)$ \\
\hline Violencia sexual & $65(31)$ & $31(14.8)$ \\
\hline
\end{tabular}

A fin de clarificar la posible relación de los factores seleccionados con la VP en HH diagnosticados con $\mathrm{VIH}$, se conformaron dos grupos extremos de comparación: los hombres sin violencia y los hombres con violencia, tanto ejercida como recibida (de acuerdo con los puntajes de la Escala de Conductas 
de Violencia del Cuestionario de Violencia Doméstica para Hombres Gay, de Rodríguez y Toro (2005) (Tabla 3).

Tabla 3. Distribución por grupos con y sin VP.

\begin{tabular}{|l|r|}
\hline \multicolumn{1}{|c|}{ Distribución de violencia } & $\boldsymbol{n}(\mathbf{\%})$ \\
\hline Sin violencia & $27(12.86)$ \\
\hline Solo ha recibido conductas de violencia & $28(13.33)$ \\
\hline Solo ha ejercido conductas de violencia & $8(3.81)$ \\
\hline Ha recibido y ejercido conductas de violencia & $147(70)$ \\
\hline
\end{tabular}

Para este análisis, se incluyeron los datos de 174 de los hombres encuestados (27 sin violencia y 147 con violencia recibida y ejercida), corroborando los criterios de normalidad para el grupo sin violen- cia. A continuación se presentan los descriptivos y los estadígrafos únicamente para las variables que resultaron ser diferentes entre estos grupos.

En la comparación de factores individuales, se encontraron diferencias significativas en las creencias distorsionadas en relación a la pareja y el uso de violencia, en el uso de estrategias tanto asertivas como agresivas para resolver problemas, y en el consumo de alcohol en exceso, siendo más altos los puntajes en el grupo con violencia. En los factores de la relación de pareja, las diferencias significativas se encontraron en el nivel de importancia asignada a la relación (más alto en el grupo sin violencia), así como en la percepción de violencia tanto recibida como ejercida (más alta en el grupo con violencia) (Tabla 4).

Tabla 4. Comparación de factores individuales y de relación entre HH seropositivos con y sin VP.

\begin{tabular}{|c|c|c|c|c|c|c|}
\hline & \multirow{3}{*}{$\begin{array}{c}\text { Total } \\
(\mathrm{n}=174)\end{array}$} & \multirow{3}{*}{$\begin{array}{l}\text { Sin VP } \\
(n=27)\end{array}$} & \multirow{3}{*}{$\begin{array}{c}\text { Con VP recibida } \\
\text { y ejercida } \\
(n=147) \\
\end{array}$} & \multirow{4}{*}{ gl } & \multirow{4}{*}{$\mathbf{F}$} & \multirow{4}{*}{ Sig. } \\
\hline & & & & & & \\
\hline & & & & & & \\
\hline FACTORES & M (D.E.) & M (D.E.) & M (D.E.) & & & \\
\hline Nivel de importancia de la relación & $8.77(1.56)$ & $9.33(1.14)$ & $8.67(1.61)$ & 1 & 4.22 & .041 \\
\hline Percepción de violencia recibida & $1.35(.47)$ & $1.07(.26)$ & $1.4(.49)$ & 1 & 11.3 & .001 \\
\hline Percepción de violencia ejercida & $1.26(.44)$ & $1.04(.19)$ & $1.31(.46)$ & 1 & 8.82 & .003 \\
\hline Agresividad & $7.6(6.64)$ & $.96(3.44)$ & $8.84(6.36)$ & 1 & 39.12 & .001 \\
\hline Asertividad & $11.42(7.08)$ & $3.37(5.51)$ & $12.9(6.31)$ & 1 & 53.8 & .001 \\
\hline Distorsiones cognitivas & $35.52(10.8)$ & $29.67(12.06)$ & $36.6(10.24)$ & 1 & 9.86 & .002 \\
\hline Consumo excesivo de alcohol & $1.64(.48)$ & $1.48(.51)$ & $1.67(.47)$ & 1 & 3.7 & .056 \\
\hline
\end{tabular}

En general, las puntuaciones de agresividad y asertividad en la Escala de Solución de Conflictos del Cuestionario de Violencia Doméstica para Hombres Gay fueron bajas, hallándose diferencias significativas entre los grupos extremos sujetos a análisis; el grupo con violencia recibida y ejercida fue el que alcanzó puntuaciones más altas en ambas estrategias, teniendo en cuenta que la escala evalúa la frecuencia con que se utilizan estas últimas, y no así el nivel o la efectividad de los estilos de afrontamiento que manejan, las puntuaciones estarían indicando una mayor ocurrencia de conflictos en este grupo, lo que le lleva a utilizar con mayor frecuencia ambas estrategias.

En los factores de estrés de minoría se encontraron diferencias significativas en la apertura sobre la orientación sexual (outness) con la familia, los amigos LGBT y el personal de salud; en todos los casos hubo una mayor apertura en el grupo con VP recibida y ejercida. En este mismo grupo hubo puntajes más altos de afirmación gay y puntajes más bajos de homonegatividad internalizada (Tabla 5).

Al comparar la historia de abuso intergeneracional, hubo diferencias significativas en el abuso sexual por parte del padre; maltrato emocional hacia la pareja e hijos, y físico hacia los hijos por parte de la madre; maltrato emocional y abuso sexual por parte de los hermanos, y maltrato emocional de alguna otra persona de la familia (Tabla 6). 
Tabla 5. Comparación de factores de estrés de minoría entre HH seropositivos con y sin VP.

\begin{tabular}{|l|r|r|r|r|r|}
\cline { 2 - 6 } \multicolumn{1}{c|}{} & \multicolumn{1}{c|}{$\begin{array}{c}\text { Sin VP } \\
(\mathbf{n}=\mathbf{2 7})\end{array}$} & $\begin{array}{c}\text { Con VP recibida } \\
\text { y ejercida } \\
(\mathbf{n}=\mathbf{1 4 7})\end{array}$ & gl & F & \multirow{2}{*}{ Sig. } \\
\hline \multicolumn{1}{|c|}{ FACTORES } & M (D.E.) & M (D.E.) & & & \\
\hline Outness familia & $1.56(1.12)$ & $2.08(.92)$ & 1 & 6.88 & .009 \\
\hline Outness amigos LGBT & $2(1.03)$ & $2.46(.79)$ & 1 & 6.96 & .009 \\
\hline Outness personal de salud & $1.78(1.28)$ & $2.22(.99)$ & 1 & 4.41 & .045 \\
\hline Afirmación gay & $20.26(11.55)$ & $30.27(8.98)$ & 1 & 25.78 & .001 \\
\hline Homonegatividad internalizada & $53.33(13.47)$ & $45.05(16.34)$ & 1 & 6.15 & .014 \\
\hline
\end{tabular}

Al comparar la historia de abuso intergeneracional, hubo diferencias significativas en el abuso sexual por parte del padre; maltrato emocional hacia la pareja e hijos, y físico hacia los hijos por parte de la madre; maltrato emocional y abuso sexual por parte de los hermanos, y maltrato emocional de alguna otra persona de la familia (Tabla 6).

Tabla 6. Comparación de historia de abuso intergeneracional entre HH seropositivos con y sin VP.

\begin{tabular}{|l|r|r|r|r|r|r|}
\cline { 2 - 7 } \multicolumn{1}{c|}{} & \multicolumn{1}{c|}{$\begin{array}{c}\text { Total } \\
\text { (n= 174) }\end{array}$} & $\begin{array}{c}\text { Sin VP } \\
\mathbf{( n = 2 7 )}\end{array}$ & $\begin{array}{c}\text { Con VP } \\
\text { recibida y } \\
\text { ejercida } \\
\mathbf{( n = 1 4 7 )}\end{array}$ & gl & F & Sig. \\
\hline \multicolumn{1}{|c|}{ PERSONA Y TIPO DE ABUSO } & M (D.E.) & M (D.E.) & M (D.E.) & & & \\
\hline Padre abusó sexualmente de sus hijos. & $0.91(.37)$ & $.81(.55)$ & $.93(.33)$ & 1 & 7.83 & .006 \\
\hline Madre violentó físicamente a sus hijos. & $1.23(.53)$ & $.96(.43)$ & $1.28(.53)$ & 1 & 8.41 & .004 \\
\hline Madre maltrató emocionalmente a su pareja. & $1.14(.59)$ & $.89(.32)$ & $1.18(.62)$ & 1 & 5.8 & .017 \\
\hline Madre maltrató emocionalmente a sus hijos. & $1.13(.49)$ & $.96(.33)$ & $1.16(.51)$ & 1 & 3.83 & .052 \\
\hline Hubo maltrato emocional entre hermanos. & $1.2(.61)$ & $1.15(.36)$ & $1.21(.64)$ & 1 & 9.86 & .002 \\
\hline Hermanos abusaron sexualmente de usted. & $1(.30)$ & $1.15(.36)$ & $.97(.28)$ & 1 & 7.81 & .006 \\
\hline Alguna otra persona lo maltrató e mocionalmente. & $1.45(.51)$ & $1.19(.39)$ & $1.5(.51)$ & 1 & 9.27 & .003 \\
\hline
\end{tabular}

\section{DISCUSIÓN}

Los resultados obtenidos en la presente investigación muestran que un alto porcentaje de los hombres encuestados había experimentado alguna conducta de violencia en su relación de pareja $(83.8 \%$ había recibido alguna conducta violenta y $74.3 \%$ había ejercido alguna), y que la frecuencia de conductas violentas en la pareja fue más alta que la que se ha reportado en parejas de hombres en otros países (McClennen, 2005; Pantalone et al., 2014; Pantalone et al., 2012; Pimentel, Cheng y Kelly, 2015; Tran et al., 2014).

A pesar de que poco más de $80 \%$ de los participantes había recibido alguna conducta violenta de parte de su pareja, solo $29.5 \%$ consideró que había sido víctima, y aunque casi $75 \%$ de los hom- bres entrevistados había ejercido alguna conducta violenta hacia su pareja, únicamente $22 \%$ pensaba que su pareja había sido víctima de violencia en su relación. Tal discrepancia se ha observado también en estudios sobre la violencia en el noviazgo en adolescentes y adultos jóvenes (De la Villa, García, Cuetos y Sirvent, 2017) y contra la mujer (Menéndez, Pérez y Lorence, 2013), en los que se ha establecido la distinción entre el maltrato declarado (en el que la persona tiene conciencia del abuso y manifiesta haber recibido violencia) y el maltrato técnico (en el que están presentes las conductas de violencia identificadas a partir de las respuestas a un listado de situaciones, pero de las que la persona no tiene conciencia).

Esto deja ver cómo algunas conductas violentas se normalizan en el contexto de la relación 
de pareja (lo que posiblemente está asociado a creencias distorsionadas), por lo cual en muchas ocasiones el individuo no identifica que dichas conductas constituyen un acto de violencia, sobre todo las conductas de violencia emocional o de coerción sexual. Bringas et al. (2015) explican que en muchas ocasiones la vivencia de una experiencia violenta no está asociada al reconocimiento de la misma, en parte por la atribución de normalidad a una conducta, basada en una interpretación errónea de lo que es el afecto. Ya Loinaz (2014) ha señalado que las distorsiones cognitivas influyen en el desarrollo y mantenimiento de la VP en tanto que existe una negación, minimización y culpabilización de la víctima, lo que promueve una menor atribución de responsabilidad de parte del agresor.

Al comparar factores psicosociales que se han asociado con la VP entre los grupos sin conductas de violencia, y en aquellos hombres con conductas de violencia recibida y ejercida, se encontraron diferencias significativas en el nivel de importancia asignado a la relación, siendo mayor dicho nivel en el grupo sin conductas de violencia; de igual manera, la percepción de violencia tanto recibida como ejercida fue menor en este grupo. Estos datos son congruentes con lo sugerido por Muñoz, González, Fernández y Fernández (2015), quienes señalan que cuando hay una mayor satisfacción con la relación -y por lo tanto se le asigna una mayor importancia- hay un menor número de conflictos y menos riesgo de manifestar conductas de violencia dentro de la pareja, aunque dichos autores lo establecen principalmente al contexto de las relaciones heterosexuales.

En concordancia con lo que se ha reportado en otros estudios sobre VP, tanto en parejas heterosexuales (Ferrer et al., 2006) como homosexuales (Siemieniuk et al., 2013), y corroborando la hipótesis planteada al inicio de este análisis, las distorsiones cognitivas en la pareja y el uso de violencia en su relación se hallaron con mayor frecuencia en el grupo de hombres con violencia recibida y ejercida, en contraste con aquellos sin VP. Minimizar las conductas de violencia, culpabilizar a quien las recibe y atribuir menor responsabilidad a quien las ejerce fueron las distorsiones ocurridas con mayor frecuencia. Otros estudios han documentado que este tipo de distorsiones influyen en el desarrollo y mantenimiento de la violencia (Loinaz, 2014; Siemieniuk et al., 2013); por lo tanto, es necesario no solamente intervenir en el desarrollo de habilidades conductuales, sino también en el cambio de estas creencias acerca de la pareja.

Por otra parte, el uso o abuso de alcohol y otras drogas se ha identificado como un factor de riesgo de VP en parejas heterosexuales (Muñoz et al., 2 015) como homosexuales (Edwards et al., 2015). Más de $60 \%$ de los participantes en este estudio habían consumido alcohol en exceso, y cerca de la mitad alguna droga. El consumo de alcohol en exceso se apreció de manera ligeramente más alta en el grupo de hombres con violencia recibida y ejercida; sin embargo, no se evaluó la frecuencia de este consumo, y la mayoría de los participantes reportaron no estar bajo los efectos del alcohol cuando ocurrieron situaciones de VP.

Tal como se reporta en la literatura, los hombres en el grupo con violencia recibida y ejercida expresaron una mayor frecuencia de antecedentes de violencia en su familia de origen (Pantalone et al., 2012; Rodríguez y Toro, 2005). Dentro de las conductas evaluadas, reportaron con más frecuencia que el padre había abusado sexualmente de sus hijos, así como haber sufrido la violencia física y emocional ejercida por la madre (tanto a los hijos como a la pareja). Esta información coincide con lo hallado por autores que sostienen que la exposición a comportamientos violentos en la familia de origen genera un repertorio de conductas violentas y un sistema de creencias que posteriormente se replican en las relaciones amorosas, ya homosexuales (Barrientos, Rodríguez, Escartín y Longares, 2016; Chong, Mak y Kwong, 2013), ya heterosexuales (Dardis, Dixon, Edwards y Turchik, 2015; Muñoz et al., 2 015).

Retomando el marco socioecológico del estrés de minorías, dos factores que pueden relacionarse con el estrés por pertenecer a una minoría sexual son la homonegatividad internalizada y la apertura sobre la orientación sexual. De hecho, se encontraron diferencias estadísticamente significativas entre los grupos comparados en el nivel de apertura (outness) con la familia, con las amistades LGBT y con el personal de salud. Contrariamente a lo esperado, el grupo con violencia recibida y ejercida alcanzó puntuaciones más altas 
de outness (que indican una mayor apertura) con personas de esos tres ámbitos. De igual manera, este grupo obtuvo puntuaciones más altas de afirmación gay y más bajas de homonegatividad internalizada.

Los datos aquí obtenidos indican la posibilidad de que los hombres con mayor apertura sobre su orientación sexual y que valoran su homosexualidad como una parte positiva e importante de su vida, sean más susceptibles a recibir rechazo y violencia por parte de las personas más cercanas (familiares y pares), así como de las instituciones de salud. De esta manera, factores individuales que pudieran considerarse positivos (apertura y afirmación) los exponen a situaciones sociales y comunitarias de discriminación y maltrato que podrían aumentar su riesgo de VP.

Aunque en la presente investigación se encontraron altos porcentajes de conductas de VP recibida y ejercida, es necesario señalar que las respuestas de los participantes fueron categorizadas según la presencia o ausencia de violencia, sin especificar la frecuencia e intensidad de dichas conductas. Este y otros estudios ejemplifican la falta de consenso que todavía existe para definir operacionalmente y medir adecuadamente el fenómeno de VP (Finneran, Chard, Sineath, Sullivan y Stephenson, 2012; Rodríguez y Toro, 2005; Wall, Sullivan, Kleinbaum y Stephenson, 2014), por lo que se requieren esfuerzos colaborativos entre los diferentes actores involucrados en el estudio de la VP para sistematizar eventualmente su medición en sus diferentes categorías.

El uso de instrumentos de autorreporte constituye otra limitación importante en el presente estudio, toda vez que puede haber sesgos de memoria, deseabilidad social o algunos otros factores que interfieran al contestar los cuestionarios (tiempos prolongados de espera, cansancio, necesidad de terminar pronto, etc.). Empero, las características propias del fenómeno estudiado dificultan la utilización de otros medios en muestras grandes (como registros u observaciones conductuales), en tanto que las escalas aquí utilizadas mostraron propiedades psicométricas adecuadas para medir los conceptos evaluados.

Cabe mencionar que el escenario donde se llevó a cabo la presente investigación es una clíni- ca con características muy particulares dentro de los servicios de salud que se brindan en la Ciudad de México, y que los hombres que se atienden ahí también muestran un perfil sociodemográfico que puede diferir del de quienes asisten a otros centros de detección y atención a personas con VIH. Por lo tanto, la generalización de los resultados aquí obtenidos debe hacerse con cautela, a la vez que se sugieren mayores esfuerzos para su replicación en otros contextos, lo que ayudaría además a tener una muestra más amplia que permita observar una tendencia más clara del comportamiento de los datos y posibilite la realización de análisis estadísticos posteriores con los que puedan establecerse modelos predictivos y explicativos más complejos. Considerando que aquí no se hallaron diferencias significativas en las variables relacionadas con el estatus de VIH, valdría la pena incluir en futuras investigaciones hombres homosexuales con y sin diagnóstico confirmado.

A pesar de las limitaciones mencionadas, el presente estudio constituye uno de los primeros acercamientos para conocer y describir el fenómeno de la VP entre HH con VIH en México. Los resultados aquí obtenidos permiten corroborar algunos factores individuales que se han asociado a la ocurrencia de conductas de VP, tanto en parejas heterosexuales como en aquellas del mismo sexo; pero además dejan ver cómo es que otros factores propios de pertenecer a una minoría sexual, como la homonegatividad internalizada y la apertura sobre la orientación sexual, pudieran exponer a estos hombres a situaciones de VP resultantes del rechazo y la violencia social a la que están expuestos, especialmente de la familia, los pares y el personal de salud.

Los datos aquí presentados señalan la importancia de comprender el fenómeno de la violencia de pareja entre hombres homosexuales con VIH como un fenómeno complejo y multidimensional. Se puede hablar de una relación violenta en la que hay implicados factores conductuales, cognitivos, sociales y propios de la relación que requieren ser identificados y modificados; de igual manera, siguiendo el modelo socioecológico de estrés de minorías, se evidencia que es necesario el desarrollo de intervenciones individuales, familiares y comunitarias específicas. 
Así, las intervenciones individuales en el futuro deberán estar diseñadas para aprender a identificar conductas de violencia en la relación de pareja, desarrollar habilidades de comunicación asertiva y de solución de conflictos y modificar falsas creencias sobre la pareja y el uso de la violencia, pero además será necesario desarrollar in- tervenciones colectivas, sociales o comunitarias para disminuir el estigma y la violencia hacia la población homosexual, particularmente hacia una población como la señalada, para comenzar a visibilizar la problemática que también existe en estas parejas al promover la detección y la atención oportuna de dicha situación.

Citación: Alderete A., C., Robles G., R., Ramos L., Luciana., Benjet, C., Alvarado S., N. y Lazcano R., M. (2021). Factores psicosociales asociados con la violencia de pareja entre hombres homosexuales mexicanos con VIH. Psicología y Salud, 31(2), 255-265. https://doi.org/10.25009/ pys.v31i2.2694.

\section{REFERENCIAS}

Alderete A., C., Robles G., R., Vega R., H., Ramos L., L., Benjet, C. y Lazcano R., M. (2017, julio). Propiedades psicométricas del inventario de homonegatividad internalizada en hombres mexicanos que tienen sexo con hombres. Memorias del XXXVI Congreso Interamericano de Psicología. Mérida, Yuc., México.

Ard, K.L. y Makadon, H.J. (2011). Addressing intimate partner violence in lesbian, gay, bisexual, and transgender patients. Journal of General Internal Medicine, 26, 930-933.

Badenes R., L., Sánchez M., J. y Longobardi, C. (2019). The relationship between internalized homophobia and intimate partner violence in same-sex relationships: A meta-analysis. Trauma, Violence \& Abuse, 20(3), 331-343.

Barrientos, J., Rodríguez C., A., Escartín. J. y Longares, L. (2016). Violencia en parejas del mismo sexo: revisión y perspectivas actuales. Revista Argentina de Clínica Psicológica, 25(3), 289-298.

Bringas M., C., Cortés A., L., Antuña B., M.A., Flores G., M., López C., J. y Rodríguez D., F.J. (2015). Análisis diferencial de la percepción de jóvenes sobre maltrato en el noviazgo. Revista Latinoamericana de Ciencias Sociales, Niñez y Juventud, 13(2), 737-748.

Chong, E., Mak, W. y Kwong, M. (2013). Risk and protective factors of same-sex intimate partner violence in Hong Kong. Journal of Interpersonal Violence, 28(7), 1476-1497.

Dardis, C.M., Dixon, K.J., Edwards, K.M. y Turchik, J.A. (2015). An examination of the factors related to dating violence perpetration among young men and women and associated theoretical explanations: A review of the literature. Trauma, Violence \& Abuse, 16(2), 136-152.

De la Villa M., M., García, A., Cuetos, G. y Sirvent, C. (2017). Violencia en el noviazgo, dependencia emocional y autoestima en adolescentes y jóvenes españoles. Revista Iberoamericana de Psicología y Salud, 8(2), 96-107.

Echeburúa, E. y Fernández M., J. (1998). Hombres maltratadores. En E. Echeburúa y P. Corral (Eds.): Manual de violencia familiar (pp. 73-175). Madrid: Siglo XXI.

Edwards, K.M., Sylaska, K.M. y Neal, A.M. (2015). Intimate partner violence among sexual minority populations: A critical review of the literature and agenda for future research. Psychology of Violence, 5(2), 112-121.

Ferrer P., V.A., Bosch F., E., Ramis P., M.C., Torres E., G. y Navarro G., C. (2006). La violencia contra las mujeres en la pareja: creencias y actitudes en estudiantes universitarios/as. Psicothema, 18(3), 359-366.

Finneran, C., Chard, A., Sineath, C., Sullivan P. y Stephenson, R. (2012). Intimate partner violence and social pressure among gay men in six different countries. West Journal of Emergency Medicine, 13(3), 260-271.

Frost, D. y Meyer, I. (2009). Internalized homophobia and relationship quality among lesbians, gay men and bisexuals. Journal of Counselling Psychology, 56(1), 97-109.

Graham, L., Jensen, T., Givens, A., Bowen, G. y Rizo, C. (2019). Intimate partner violence among same-sex couples in college: A propensity score analysis. Journal of Interpersonal Violence, 34(8), 1583-1610.

Kimmes, J., Mallory, A., Spencer, C., Beck, A., Cafferky, B. y Stith, S. (2019). A meta-analysis of risk markers for intimate partner violence in same-sex relationships. Trauma, Violence \& Abuse, 20(3), 374-384.

Kubicek, K. (2018). Setting an agenda to address intimate partner violence among young men who have sex with men: A conceptual model and review. Trauma, Violence \& Abuse, 19(4), 473-487.

Loinaz, I. (2014). Distorsiones cognitivas en agresores de pareja: Análisis de una herramienta de evaluación. Terapia Psicológica, $32(1), 5-17$. 
Mayfield, W. (2001). The development of an internalized homonegativity inventory for gay men. Journal of Homosexuality, 41(2), 53-76.

McClennen, J.C. (2005). Domestic violence between same-gender partners. Journal of Interpersonal Violence, 20(2), 149-154.

McClennen, J.C., Summers, A.B. y Vaughan, C. (2002). Gay men's domestic violence: Dynamics, help-seeking behaviors, and correlates. Journal of Gay and Lesbian Social Services, 14(1), 23-49.

Menéndez Á.D., S., Pérez P., J. y Lorence L., B. (2013). La violencia de pareja contra la mujer en España: Cuantificación y caracterización del problema, las víctimas, los agresores, y el contexto social y profesional. Psychosocial Intervention, 22, 41-53.

Muñoz R., M., González L., P., Fernández G., L. y Fernández R., S. (2015) Violencia en el noviazgo: Realidad y prevención. Madrid: Ediciones Pirámide.

Ortega, A. (2014). Agresión en parejas homosexuales en España y Argentina: Prevalencias y heterosexismo. Tesis inédita de doctorado. Madrid: Universidad Complutense de Madrid.

Pantalone, D., Rood, B., Morris, B. y Simoni, J. (2014). A systematic review of the frequency and correlates of partner abuse in HIV-infected women and men who partner with men. Journal of the Association of Nurses in AIDS Care, 25, S15-S35.

Pantalone, D., Schneider, K., Valentine, S. y Simoni, J. (2012). Investigating partner abuse among HIV-positive men who have sex with men. AIDS and Behavior, 16(4), 1031-1043.

Peterman, L.M. y Dixon, C.G. (2003). Intimate partner abuse between same-sex partners: Implications for counseling. Journal of Counseling and Development, 81, 40-59.

Pimentel, M.L., Cheng, A.L. y Kelly, P.J. (2015). Syndemic theory and male same sex intimate partner violence: An urban/ non-urban comparison. Open Access Library Journal, 2, e1407.

Ramachandran, S., Yonas, M., Silvestre, A. y Burke, J. (2010). Intimate partner violence among HIV-positive persons in an urban clinic. AIDS Care, 22(12), 1536-1543.

Relf, M. (2001). Battering and HIV in men who have sex with men: A critique and synthesis of the literature. Journal of the Association of Nurses in AIDS Care, 12(3), 41-48.

Reyes M., F., Rodríguez J., R. y Malavé, S. (2005). Manifestaciones de la violencia doméstica en una muestra de hombres homosexuales y mujeres lesbianas puertorriqueñas. Revista Interamericana de Psicología, 39(3), 449-456.

Roberts, J.A. (2005). An integrative review of intimate partner violence among men who have sex with men: correlates of victimization and development of a conceptual framework. Humanity and Society, 29(2), 126-136.

Rodríguez M., S. y Toro A., J. (2005). Description of a domestic violence measure for Puerto Rican gay males. Journal of Homosexuality, 50(1), 155-173.

Saldivia M., C., Faundez R., B., Sotomayor L., S. y Cea L., F. (2017). Violencia íntima en parejas jóvenes del mismo sexo en Chile. Última Década, 46, 184-212.

Schafer, K.R., Brant, J., Gupta, S., Thorpe, J., Winstead-Derlega, C., Pinkerton, R., Laughon, K., Ingersoll, K. y Dillingham, R. (2012). Intimate partner violence: A predictor of worse HIV outcomes and engagement in care. Aids Patient Care and STDS, 26(6), 356-365.

Siemieniuk, R.A.C., Miller, P., Woodman, K., Ko, K., Krentz, H.B. y Gill, M.J. (2013). Prevalence, clinical associations, and impact of intimate partner violence among HIV-infected gay and bisexual men: a population-based study. HIV Medicine, 14, 293-302.

Téllez-Santaya, P.O. y Walters, A.S. (2011). Intimate partner violence within gay male couples: Dimensionalizing partner violence among Cuban gay men. Sexuality and Culture, 15, 153-178.

Toro A., J. y Rodríguez M., S. (2001). Violencia doméstica en parejas de hombres gay puertorriqueños: prevalencia, violencia intergeneracional, conductas adictivas y destrezas de manejo de conflictos. Perspectivas Psicológicas, 3(4), 164-172.

Tran, A., Lin, L., Nehl, E., Talley, C., Dunkle, K. y Wong, F. (2014). Prevalence of substance use and intimate partner violence in a sample of A/PI MSM. Journal of Interpersonal Violence, 29(11), 2054-2067.

Wall, K.M., Sullivan, P.S., Kleinbaum, D. y Stephenson, R. (2014). Actor-partner effects associated with experiencing intimate partner violence or coercion among male couples enrolled in an HIV prevention trial. BMC Public Health, 14, $209-221$. 